\title{
Study on Dynamics Identification of the Foot Viscoelasticity of a Humanoid Robot
}

\author{
Yuya Mikami*, Thomas Moulard** \\ Eiichi Yoshida**, Gentiane Venture* \\ *Department of Mechanical Systems Engineering, Tokyo University of Agriculture and Technology, 2-24-16 Nakacho \\ Koganei, Japan (e-mail:venture@cc.tuat.ac.jp). \\ **CNRS-AIST, JRL (joint Robotics Laboratory), UMI 3218/CRT, Intelligent Systems Research Institute, AIST Central 2, 1-1-1 \\ Umezono Tsukuba Ibaraki, Japan (e-mail: thomas.moulard@aist.go.jp, e.yoshida@aist.go.jp).
}

\begin{abstract}
This paper describes the dynamics identification of the foot viscoelasticity of humanoid robots. It is important to know correctly dynamics parameters of link and joint which constitute a robot for its simulation and model based control. When walking, humanoids receive impulsive force at each step. Some humanoids have shock absorber and rubber bush in their soles. In simulation, accuracy of inertial parameters and viscoelastic parameters that compose robot model is important to simulate their motion accurately. We identify the viscoelastic parameters of HRP-2 using the base-link dynamics and viscoelastic joint dynamics. We modeled the humanoid robot HRP-2 sole rubber bush with a simple structure and identify the viscoelastic parameters with parallel model that is composed of elasticity and viscosity and offset torque. We finally validate the identified parameters with some additional motions.
\end{abstract}

\section{INTRODUCTION}

It is important to know correctly the robot's parameters for its control. For example, computed torque method (Siciliano, Khatib, 2008) is usually used to control a robot and this method requires a precise dynamic model of the robot. However dynamic modeling of the robot using CAD data usually includes undesirable error as it often does not take into account the wiring. Then, it is required to identify these parameters with robot motion. The classic identification of the inertial parameters of robots uses the joint torques, or their estimation. However this method is affected by factors such as joint friction, elasticity and viscosity disturbance. Therefore, the base-link identification method presented in (Ayusawa et $a l ., 2008$ ) which does not require the torque measurements is useful to identify the dynamics of complex systems, in particular humanoid robots (Iwasaki et al., 2012).

In a two-legged robot, since the sole parts are the main junction points with the environment, they play an important role when operating the robot. When walking, humanoids receive impulsive force at each step. To avoid undesirable dynamics effects, shock absorbers and rubber bushes, that create a complex structure, are used in their soles. This structure must be simulated in the robot dynamic simulator to achieve realistic simulations and model based control. The accuracy of inertial parameters and the viscoelastic parameters specific to the robot are crucial to simulate the robot motions accurately. In this research, after identifying the inertial parameters (Iwasaki et al., 2012), we now identify the viscoelastic properties of the sole rubber bush of the humanoid robot HRP-2.

In this paper, we propose and apply a method to identify the joint viscoelastic parameters of legged systems using the base-link dynamics and viscoelastic joint dynamics of the foot without using dedicated displacement sensors. We mod- eled HRP-2 sole rubber bush viscoelasticity and identified its viscoelastic parameters using motion data generated for this purpose. We finally validate the identified parameters with several additional motions.

\section{IDENTIFICATION OF THE ROBOT JOINT VISCO- ELASTICITY}

The equation of motion of a moving robot is composed of the matrix of link mass, the tensor of inertia, the center of mass, the joint angles, velocity and acceleration of the kinematic chain and the acceleration of the root link (Yoshida, et al., 1995). From the robot equation of motion, the identification model can be written as eq.1. Here we propose to decompose the vector of parameters to identify into the inertial parameters contribution $\phi_{I}$ and the joint contribution $\phi_{J}$, compared to the identification model introduced in classic literature (Kawasaki, et al., 1991, Ayusawa et al., 2008, Siciliano, Khatib, 2008).

$$
\left[\begin{array}{cc}
Y_{B I} & 0 \\
Y_{J I} & Y_{J J}
\end{array}\right]\left[\begin{array}{l}
\phi_{I} \\
\phi_{J}
\end{array}\right]=\left[\begin{array}{l}
0 \\
\tau
\end{array}\right]+\sum_{k=1}^{N_{C}}\left[\begin{array}{c}
K_{B k} \\
K_{J k}
\end{array}\right] F_{k}
$$

where:

$$
\begin{array}{ll}
n & : \text { Number of links. } \\
N_{I} & : \text { Number of inertial parameters of the robot. } \\
N_{J} & : \text { Number of joint parameters of the robot. }
\end{array}
$$

$Y_{B I}$ : : Observation matrices of the robot motion. These

$Y_{J}, \quad$ matrices are function of joint angle, velocity and $Y_{J J} \quad$ acceleration and reflect inertial parameters such as mass, tensor of inertia and center of mass to baselink forces and torques. $Y_{B I}$ is a $6 \times N_{I}$ matrix. This matrix translates inertial parameters to base-link 
generalized forces. $Y_{J I}$ is a $n \times N_{I}$ matrix. This matrix translates inertial parameters to joint torques. $Y_{J J}$ is a $n \times N_{J}$ matrix. This matrix translates joint parameters to joint torques and changes with the viscoelasticity models of a joint.

$\phi_{I} \quad:$ All inertial parameters of the robot. This vector contains all link mass, tensor of inertia and center of mass as follows:

$\phi_{I}=\left[\phi_{1} \cdots \phi_{n}\right]^{T}$

$\phi_{i}=\left[m_{i} m s_{x i} m s_{y i} m s_{z i} I_{x x i} I_{y y i} I_{z z i} I_{x y i} I_{y z i} I_{z x i}\right]^{T}$ where:

$m_{i}[\mathrm{~kg}]$ is the mass of link $i$.

$m s_{x i}, m s_{y i}, m s_{z i}[\mathrm{~kg} \mathrm{~m}]$ are the components of the inertia tensor.

$\phi_{J} \quad$ : All joint parameters of the robot. This vector contains the elastic coefficients, the coefficients of viscosity and the coefficients of friction if they exist. This vector changes with the viscoelasticity models used to model the joint.

$\tau:$ Joint torque vector (internal and actuation torque). $\tau$ is a $n \times 1$ vector.

$N_{C} \quad$ : Number of contact point of the robot with the environment.

$K_{B k}, \quad$ : Transfer matrices of contact force to forces and

$K_{J k} \quad$ torques. $K_{B k}$ is a $6 \times 6$ matrix. This matrix translates the contact forces and the torques to forces applied

to the base-link. $K_{J k}$ is a $n \times 6$ matrix. This matrix translates contact forces and contact torques to torques applied to each links.

$F_{k} \quad$ : Contact force and torque of the robot with the environment. $F_{k}$ is a $6 \times 1$ vector.

Eq. 1 is the full motion equation of the robot; it includes inertial parameters that have no direct effect on the motion (Gautier, 1990, Gautier, et al., 1991, Kawasaki, et al., 1991). As it is preferable to have a minimal set of inertial parameters for identification, we can solve eq. 2 to obtain the inertial parameters $\phi_{I}$ through the minimal identification model and then eq. 3 using the results of eq. 2 to find the joint viscoelastic properties $\phi$.

$$
\phi_{I}=Y_{B I}^{+} \sum_{k=1}^{N_{C}} K_{B k} F_{k}
$$

where:

“+” expresses the Moore-Penrose pseudo-inverse matrix.

$$
\phi_{J}=Y_{J J}^{+}\left(\tau+\sum_{k=1}^{N_{C}} K_{J k} F_{k}-Y_{J I} \phi_{I}\right)
$$

The joint torque $\tau$ is used for calculation in eq. 3 . If this is a joint equipped with an actuator, torque is needed for calcula- tion, yet when the joint is constituted by only passive elements, such as a damper, the measurement of joint torque is unnecessary as the actuated torque is 0 . The definition of the regressor matrix $Y_{J J}$ changes with the modeling of the passive joints. Therefore, the joint parameters and regressor matrix which are defined by the spring-damper-offset terms are shown in the eq. $4-5$. Here, the offset term is an element for absorbing the influence of the zero point of the force sensor and the influence of the zero point of the joint angle. On measurement, this is required and does not influence the joint characteristics. $K$ is the elastic term of a spring. $C$ is the viscous term of a damper. $O$ is the offset term. Here the regressor matrix varies with $q$, the joint angle, $\dot{q}$, the joint angular velocity and 1 is the vector in which each element is the numerical value 1 .

$$
\begin{gathered}
\phi_{J}=\left[\begin{array}{c}
K \\
C \\
O
\end{array}\right] \\
Y_{J J}=\left[\begin{array}{lll}
q & \dot{q} & 1
\end{array}\right]
\end{gathered}
$$

where: $\phi_{J}$ is a $3 \times 1$ vector. $Y_{J J}$ is, after sampling along a movement, a number of samples $\times 3$.

\section{IDENTIFICATION OF THE ROBOT'S FOOT VISCOE- LASTICITY}

\subsection{Modelling of the rubber bush}

We used the humanoid robot HRP-2 for our experiments. The foot structure of HRP-2 is complex and consists in a combination of a rubber sole and bushes under the force sensor (Fig. 1) (Isozumi et al., 2004). This sole rubber bush is the target of the identification. We modeled the rubber bush for viscoelastic identification of the rubber bush as described in section II eq.4 and eq. 5. In this paper, the model of the rubber bush has a series structure that consists of $\mathrm{Z}$-axis translational joint, pitch and roll (David et al., 2008, Nakaoka et al., 2007). It should be noted that each link is modeled as a virtual link that has no inertia.

\subsection{Estimation of the displacement of the rubber bush}

We must estimate the rubber bush displacement for identification of the rubber bush viscoelasticity. The rubber bush of HRP-2 is constituted of a passive rubber bush and the equipment which carries out direct measurement of the displacement is not installed. Therefore, it is necessary to measure indirectly the displacement of the 3 DOF using other measuring devices. In this paper, the rubber bush displacement is estimated using the kinematics of the robot, the joint encoders and the IMU which is installed in the chest. The mimetic diagram for rubber bush displacement estimation is shown in Fig. 2.

Here, HRP-2 has either one foot grounded to the floor, thus a simple tree kinematic structure is used; or both feet grounded to the floor, then a parallel kinematic structure is used. As long as the feet are grounded without slipping on the floor, this structure does not change. Here, the rubber bush displacement is estimated using this grounding condition (at 


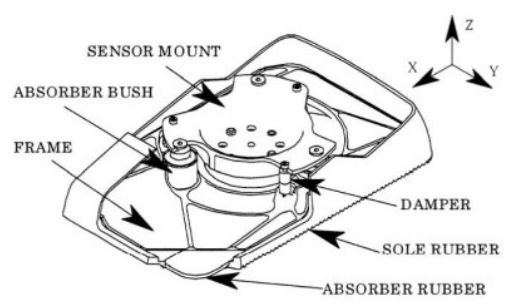

Fig. 1. Leg rubber bush design of the HRP-2(Isozumi et al., 2004)

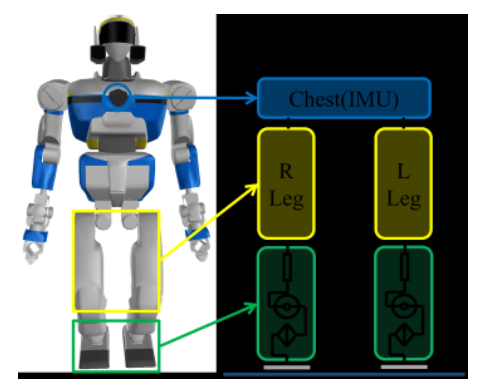

Fig. 2. Identification model of the HRP-2

least one foot in contact with ground). The roll angle and pitch angle of the rubber bush can thus be estimated. However, since the direct measurement of the chest position cannot be carried out, the $\mathrm{Z}$-axis displacement estimation of the rubber bush cannot be obtained only with the kinematics chain. Therefore, in this paper, the vertical component is removed from our model. Additional sensors are needed to estimate the $\mathrm{Z}$-axis displacement, such as accelerometers. The model of the viscoelasticity is used in order to perform viscoelasticity identification. As mentioned above, the identification type used for joint viscoelasticity is shown in eq. 6 since the elastic DOFs are passive.

$$
\phi_{J}=Y_{J J}^{+} F_{e x t}
$$

The contribution of the inertial parameter is omitted in eq. 6 . This is because the force sensor is installed on top of the rubber bush and HRP-2 can include the contribution of the inertial parameters in the force measurement directly. Moreover, $F_{\text {ext }}$ is the value acquired from the 6-axes force sensor, and we use only the data row of the roll axis and pitch-axis torque here.

\subsection{Motion generation}

The results of the identification depend highly on the motions used to solve eq. 6 (Gautier and Khalil, 1992, Swevers et al., 1997). Thus, motions that provide sufficient excitation of the DOFs considered must be generated. Moreover, in order to use the viscoelasticity identification technique proposed in this paper, as mentioned above, the contact state of the robot and the floor needs to be kept constant during the whole duration of a single motion. Yet, several motions with different contact conditions can be used to solve eq. 6 , by concatenating them. The expression for the joint parameter has the linear nature from eq. 6. Thus, motions are summarized in one expression like eq. 7 .

$$
\phi_{J}=\left[\begin{array}{c}
Y_{1}^{\text {motion }} \\
\vdots \\
Y_{m}^{\text {motion }}
\end{array}\right]^{+}\left[\begin{array}{c}
F_{1}^{\text {motion }} \\
\vdots \\
F_{m}^{\text {motion }}
\end{array}\right]
$$

where:

$m$ is a motion number. $Y_{i}^{\text {motion }}$ is a regressor matrix in motion of number $i . F_{i}^{\text {motion }}$ is the value acquired from the force sensor in motion of number $i$.

Therefore, motions which maintain a contact state and induce a sufficient rubber bush angle and velocity are required for identification. In this aim we generated several types of motions. The motions are first generated using the HRP-2 simulator. The maintaining of the balance during the whole motion is verified before being generated on the robot and then the motion are played on the actual robot.

\subsubsection{Tilting motions (6 motions)}

First, tilting motions were generated. Six motions were created from Tilt1 to Tilt6 where the two feet are always in contact with the ground. Tilt 1 to Tilt3 motions feature only horizontal displacement of the chest around an arbitrary original state using a periodic sway. The horizontal displacement amplitude is about $0.02 \mathrm{~m}$ and the period is $0.1 \mathrm{~Hz}$. The robot moves twice forward and backward, and it moves twice to the right and to the left. Moreover, 3 other tilting motions are generated. Tilt4 to Tilt6 motions, feature the same horizontal displacement as Tilt1 to Tilt3, and an additional vertical motion of the chest about $0.07 \mathrm{~m}$ and period $0.1 \mathrm{~Hz}$ simultaneously. In order to generate different types of foot rubber bush deformations, three initial states were set up: the two feet are in contact with the ground, regular standing (A), stepped toward the right leg $0.05 \mathrm{~m}$ on right-hand side (B), stepped forward of the right leg $0.1 \mathrm{~m}$ (C) (Fig. 3). To summarize the tilting motions generated are as follows:

- Tilt1 is an horizontal sway of the chest at initial state (A).

- Tilt2 is an horizontal sway of the chest at initial state (B).

- Tilt3 is an horizontal sway of the chest at initial state (C).

- Tilt4 is an horizontal and vertical sway of the chest at initial state (A).

- Tilt5 is an horizontal and vertical sway of the chest at initial state $(\mathrm{B})$.

- Tilt6 is an horizontal and vertical sway of the chest at initial state $(\mathrm{C})$.

Examples of the ZMP locus are shown in Fig. 4 for motions Tilt1 and Tilt3. It shows that the balance is kept during the whole motion: the ZMP is near the center of the support polygon in each motion.

\subsubsection{Manual sway (5 motions)}

Finally, to obtain a maximal displacement and velocity of the foot rubber bushs that would maximize excitation, manual sways of the robot are generated. Left/right manual sway and 


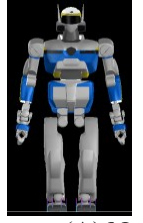

(A) Normal
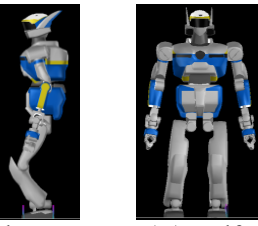

(B) Half right step

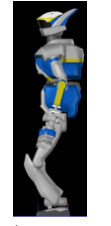

Fig. 3. The three initial states of Tilt motions

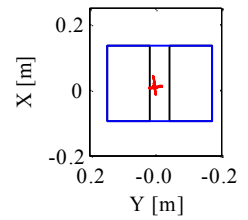

(a) Tilt1

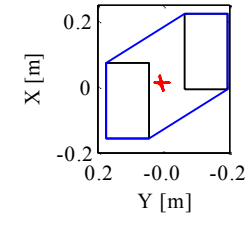

(b) Tilt3
Fig. 4. ZMP trajectories of Tilt1 (initial state (A)) and Tilt3 (initial state (C)) motions (Black: trace of the feet; Blue: support polygon; Red: the trajectory of the ZMP during the movement)

forward/backward manual sways are created from 2 initial states of the arms and 3 initial states of the legs (Fig. 5).

- Manual_right_roll raises a left leg at initial states (D \& G).

- Manual_right_pitch raises a left leg at initial state (E \& G).

- Manual_left_roll raises a left leg at initial state (D \& F).

- Manual_left_pitch raises a left leg at initial state (E \& F)

- Manual_both_pitch is initial state (E \& A).

In the above-mentioned state, an operator had a robot arm and swayed all around. The initial states are shown in Fig. 5. The ZMP locus in two case is shown in Fig. 6.

\subsubsection{Random motion (2 motions)}

In addition, in order to verify the identification results, verification motions for cross validation called Myrand were generated. Two motions, Myrand4 and Myrand5, were created. These motions consist in a sweep wave input into each joint of the upper limbs. By shaking especially the arms part with sinusoidal movements, it is expected that the resulting rubber bush displacement will become random. The motions are generated again using the HRP-2 simulator first to set joint angles, velocities and accelerations that allow an admissible joint torque.

\section{EXPERIMENTAL IDENTIFICATION RESULT}

For each of the above described motion, the rubber bush deformation is estimated in term of roll angle and pitch angle. The results are shown in Fig. 7 and Fig. 8 for motion Tilt3 and motion Manual_left_roll respectively. From these results, the rotation of the foot around the pitch and roll axes is verified. Moreover, sufficient displacement can be obtained for optimal excitation. From the eleven motions generated for identification we select a combination that provides sufficient excitation to identify each foot viscoelastic parameters: we composed Manual_right_roll and Manual_right_pitch and Manual_left_roll and Manual_left_pitch with Tilt3. And we identified the dynamics using composed motion. The obtained identification results are summarized in table 1 . The

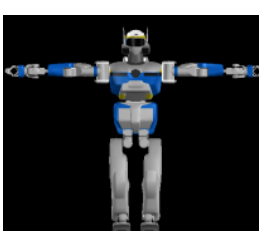

(D) Raise horizontally for roll motions

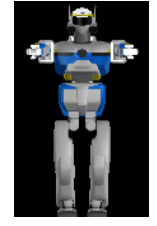

(E) Raise ahead for pitch motions

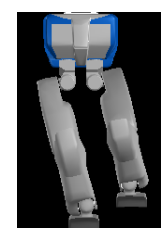

(F) Raise right leg

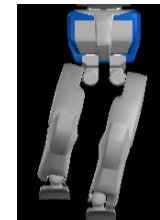

(G) Raise left leg
Fig. 5. Initial states of Manual sway motions

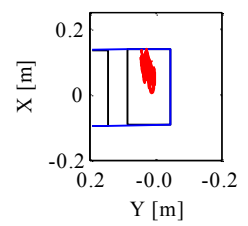

(a) Manual_right_pitch

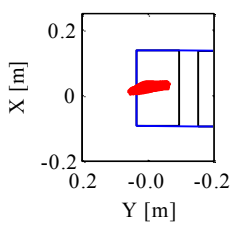

(b) Manual_left_roll
Fig. 6. ZMP trajectories of Manual_right_pitch and Manual_left_roll motions (Black: trace of the feet; Blue: support polygon; Red: the trajectory of the ZMP during the movement)

numerical value in the parenthesis following the parameter value gives the relative standard deviation (account of a percentage) of each identified parameter. Since the identification result of the viscoelasticity and an offset term have small values of relative standard deviation, we can conclude that the parameters are identified with sufficient accuracy. The direct validation presented in Fig. 9 confirms this conclusion. The sensor values of left ankle are nearly zero between 50 s to $170 \mathrm{~s}$. And also the sensor values of right ankle are nearly zero between 170 s to 350 s. This is because there is no input to the sensor for the one leg stand. In addition, we show a mean error in Fig. 9 in table2. It is thought that it is enough because the mean error is around $15 \%$ of the maximum.

Table 1. Identified parameters with Composed motion

\begin{tabular}{|c|c|c|c|c|}
\hline & Axis & $\mathrm{K}(\mathrm{Nm} / \mathrm{rad})$ & $\mathrm{C}(\mathrm{Nms} / \mathrm{rad})$ & $\mathrm{O}(\mathrm{Nm})$ \\
\hline \multirow{2}{*}{$\begin{array}{c}\text { Right } \\
\text { ankle }\end{array}$} & Pitch & $434(0.2 \%)$ & $15.89(4.2 \%)$ & $4.79(0.6 \%)$ \\
\cline { 2 - 5 } & Roll & $323(0.3 \%)$ & $17.53(2.8 \%)$ & $-0.05(38.6 \%)$ \\
\hline \multirow{2}{*}{$\begin{array}{c}\text { Left } \\
\text { ankle }\end{array}$} & Pitch & $480(0.3 \%)$ & $24.27(2.4 \%)$ & $5.66(0.6 \%)$ \\
\cline { 2 - 5 } & Roll & $352(0.3 \%)$ & $28.15(1.6 \%)$ & $-4.12(0.6 \%)$ \\
\hline
\end{tabular}

Table 2. Mean of error between direct validation and sensor

\begin{tabular}{|c|c|c|c|c|}
\hline Axis & Leg & Error & Max & $\begin{array}{c}\text { Difference with } \\
\text { the max value }\end{array}$ \\
\hline \multirow{2}{*}{ Pitch } & Right & $6.54[\mathrm{Nm}]$ & $70.1[\mathrm{Nm}]$ & $9.3[\%]$ \\
\cline { 2 - 5 } & Left & $7.54[\mathrm{Nm}]$ & $60.7[\mathrm{Nm}]$ & $12.4[\%]$ \\
\hline \multirow{2}{*}{ Roll } & Right & $3.78[\mathrm{Nm}]$ & $20.9[\mathrm{Nm}]$ & $18.1[\%]$ \\
\cline { 2 - 5 } & Left & $4.85[\mathrm{Nm}]$ & $26.7[\mathrm{Nm}]$ & $18.2[\%]$ \\
\hline
\end{tabular}

\subsection{Cross validation}

Let us now consider the validity of the parameters when it comes to reconstruct the joint torque for a motion that has not been used for identification. This is called cross validation. 

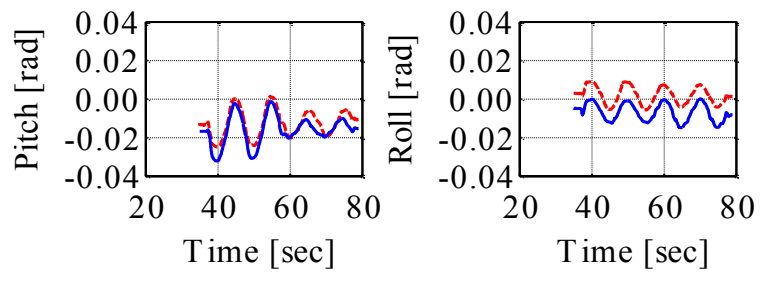

Fig. 7. Estimation results of rubber bush angles for Tilt3 (Red: right leg, Blue: left leg)
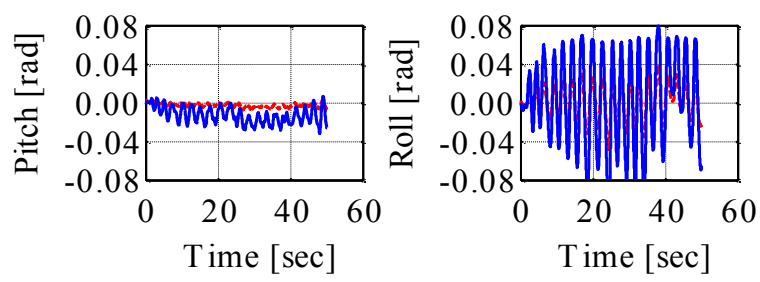

Fig. 8. Estimation results of rubber bush angles for Manual_left_roll (Red: right leg, Blue: left leg)

We reconstruct the torque produced at each axis (roll and pitch) of each foot for the motion Tilt1, Squat, Myrand4 and Manual_both_pitch from the result of the identification. Fig. 10-13 show the reconstructed torque. In addition, we give the mean error corresponding to Fig. 10-13 in table3.

Table 3. Mean of error between cross validation and sensor

\begin{tabular}{|c|c|c|c|c|c|}
\hline Axis & Leg & Tilt1 & Squat & Myrand4 & $\begin{array}{c}\text { Manual } \\
\text { both_pitch }\end{array}$ \\
\hline \multirow{3}{*}{ Pitch } & Right & $\begin{array}{c}0.79 \\
{[\mathrm{Nm}]}\end{array}$ & $\begin{array}{c}0.38 \\
{[\mathrm{Nm}]}\end{array}$ & $\begin{array}{c}2.16 \\
{[\mathrm{Nm}]}\end{array}$ & $\begin{array}{c}1.99 \\
{[\mathrm{Nm}]}\end{array}$ \\
\cline { 2 - 6 } & Left & $\begin{array}{c}0.49 \\
{[\mathrm{Nm}]}\end{array}$ & $\begin{array}{c}0.78 \\
{[\mathrm{Nm}]}\end{array}$ & $\begin{array}{c}0.83 \\
{[\mathrm{Nm}]}\end{array}$ & $\begin{array}{c}1.33 \\
{[\mathrm{Nm}]}\end{array}$ \\
\hline \multirow{3}{*}{ Roll } & Right & $\begin{array}{c}0.95 \\
{[\mathrm{Nm}]}\end{array}$ & $\begin{array}{c}0.65 \\
{[\mathrm{Nm}]}\end{array}$ & $\begin{array}{c}1.08 \\
{[\mathrm{Nm}]}\end{array}$ & $\begin{array}{c}1.35 \\
{[\mathrm{Nm}]}\end{array}$ \\
\cline { 2 - 6 } & Left & $\begin{array}{c}2.47 \\
{[\mathrm{Nm}]}\end{array}$ & $\begin{array}{c}2.39 \\
{[\mathrm{Nm}]}\end{array}$ & $\begin{array}{c}1.10 \\
{[\mathrm{Nm}]}\end{array}$ & $\begin{array}{c}2.03 \\
{[\mathrm{Nm}]}\end{array}$ \\
\hline
\end{tabular}

Although the measured torque and the reconstructed torques are generally in agreement for the pitch-axis, around the roll axis, a larger error can be seen. Two reasons can explain this larger error: firstly the smallness of the roll angle that still makes it difficult to fully identify the parameters. Secondly due to the existence of changes of adherence and of contact position due to undesirable sliding. The friction condition
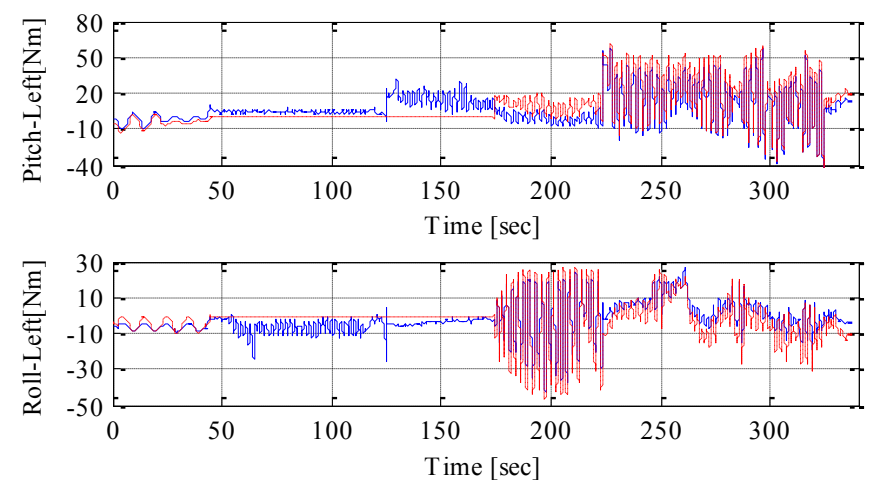

thus changes and may impact the foot viscoelasticity identification and behavior. This is a nonlinear factor generally called hysteresis. The change state in hysteresis is considered in the joint angle or the joint angular velocity as a threshold value. However, it is difficult to determine these changes in contact condition with the sensors available.

\section{CONCLUSION}

In this paper, we proposed a new method to identify the viscoelastic parameters of the feet of a humanoid robot. The method is experimentally tested on the robot HRP-2. The novelty of the proposed method lies in its usage of a sole of the humanoid as the base-link by taking advantage of the zero velocity and acceleration while the foot is fixed to the ground and to compute the rubber bushes deformation using the kinematic chain and the gyro sensor information measured in the robot's chest. The experimental results obtained are then interpreted using the relative standard deviation, direct and cross validations. The obtained results are particularly satisfying for the parameters around the pitch axis of each foot. Around the roll axis the accuracy when reconstructing the joint torque slightly decreases. This can be explained by the nonlinear behavior of the foot elasticity, the slight slipping that occurs during the movement, and the smallness of the roll despite our effort to generate enough roll angles.

These results now need to be implemented in the robot simulator and verification of the experimental behavior and the simulation behavior will be conducted.

\section{ACKNOWLEDGMENT}

The authors thank Dr. A. Kheddar from the CNRS-AIST, JRL (Joint Robotics Laboratory), UMI 3218/CRT, Intelligent Systems Research Institute, for their support for the data acquisition.

\section{REFERENCES}

Ayusawa, K., Venture, G. and Nakamura, Y. (2008). Identification of the inertial parameters of a humanoid robot using unactuated dynamics of the base-link. Proc. of the IEEE-RAS Int. Conf. on Humanoid Robots (Humanoids), 1-7.

David, A., Chardonnet, J.R., Kheddar, A., Kaneko, K., Yokoi, K. (2008). Study of an external passive shock-absorbing
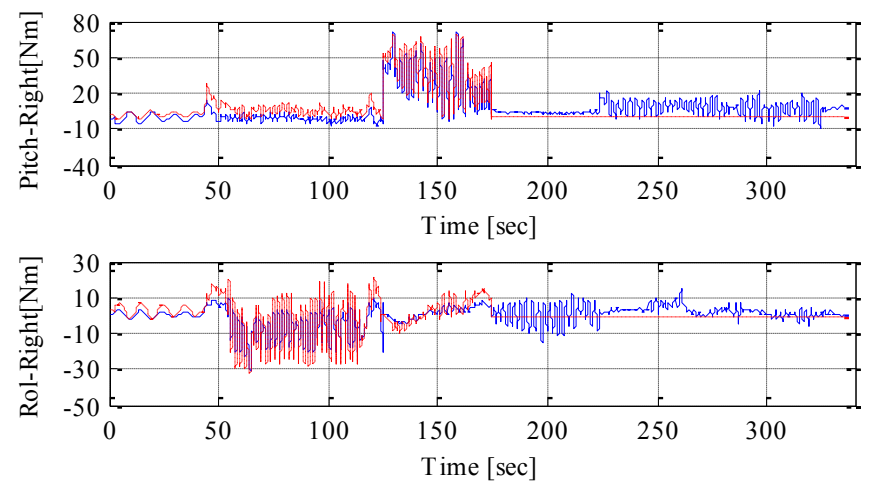

Fig. 9. Direct validation result of Composed motion (Blue: reconstructed force (direct validation), Red: sensor) 

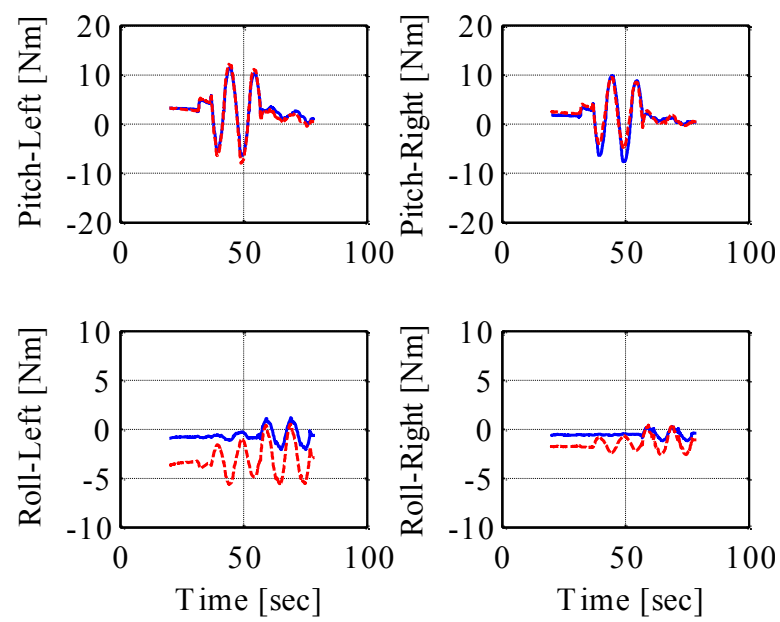

Fig. 10. Cross validation result of Tilt1 (Blue: reconstructed force (cross validation), Red: sensor)
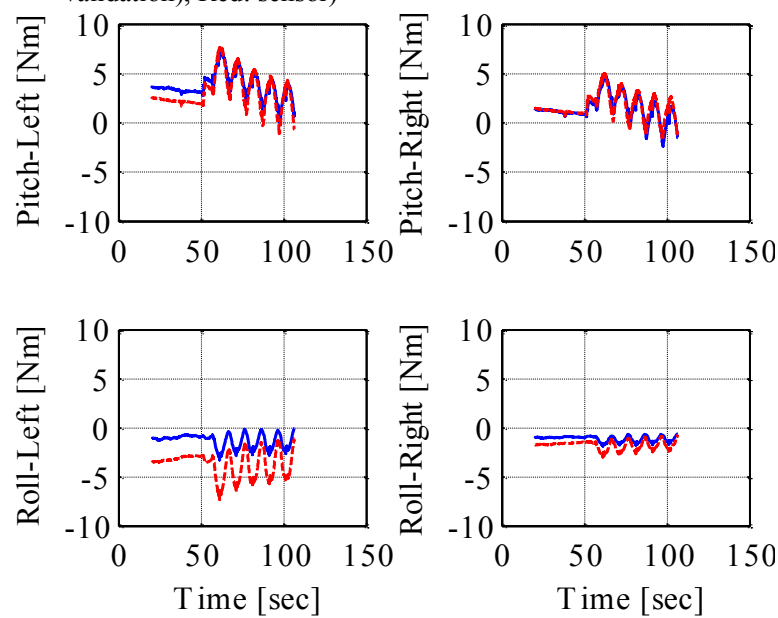

Fig. 11. Cross validation result of Squat (Blue: reconstructed force (cross validation), Red: sensor)

mechanism for walking robots. Proc. of the IEEE-RAS Int. Conf. on Humanoid Robots (Humanoids), 435-440.

Gautier, M. and Khalil, W. (1990). Direct calculation of minimum set of inertial parameters of serial robots. IEEE Trans. on Robotics and Automation, Vol. 6, no. 3, 368-373.

Gautier, M. (1991) Numerical calculation of the base inertial parameters. Journal of Robotics Systems, Vol. 8, no. 4, 485-506.

Gautier, M. and Khalil, W. (1992). Exciting trajectories for the identification of base inertial parameters of robots. The Int. Journal of Robotics Research, Vol. 11, no. 4, 362-375.

Isozumi, T., Akachi, K., Hirata, M., Kaneko, K., Kajita, S. and Hirukawa, H. (2004). Development of humanoid robot HRP-2. Journal of the Robotics Society of Japan, Vol. 22, no. 8, 1004-1012.

Iwasaki, T., Venture, G., Yoshida, E. (2012). Identification of the inertial parameters of a humanoid robot using grounded sole Link. Proc. of the IEEE-RAS Int.
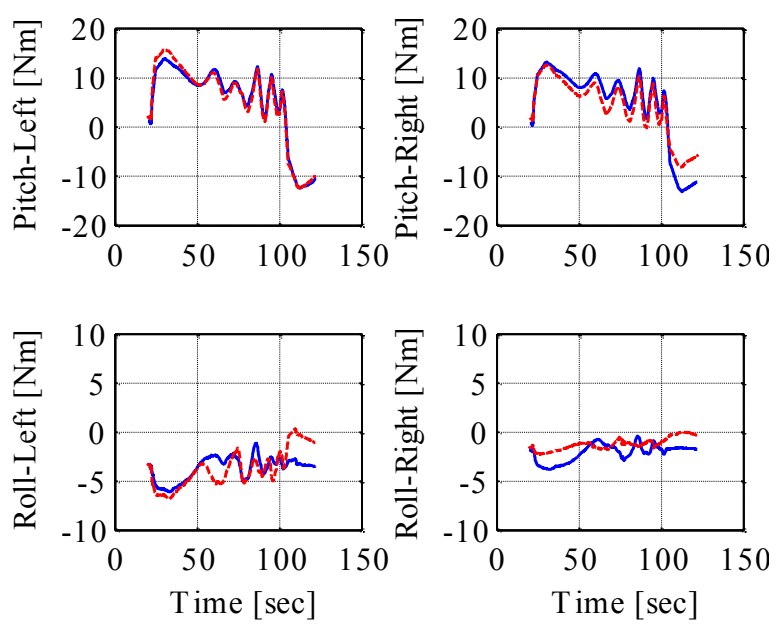

Fig. 12. Cross validation result of Myrand4 (Blue: reconstructed force (cross validation), Red: sensor)
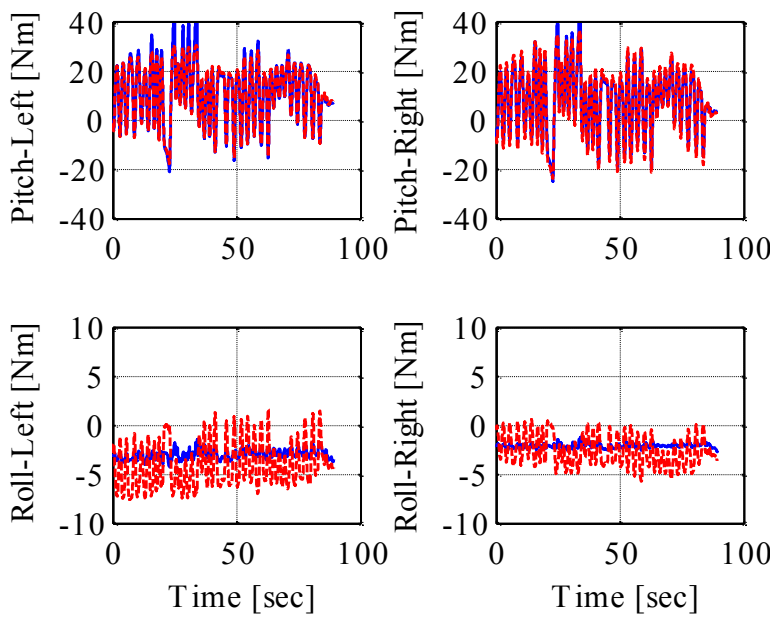

Fig. 13. Cross validation result of Manual_both_pitch (Blue: reconstructed force (cross validation), Red: sensor)

Conf. on Humanoid Robots (Humanoids), 249-254.

Kawasaki, H., Beniya, Y. and Kanzaki, K. (1991). Minimum dynamics parameters of tree structure robot models. In Int. Conf. of Industrial Electronics, Control and Instrumentation, Vol. 2, 1100-1105.

Nakaoka, S., Hattori, S., Kanehiro, F., Kajita, S., Hirukata, H., (2007). Constraint-based dynamics simulator for humanoid robots with shock absorbing mechanisms. Proc. of the Intelligent Robots and Systems, 3641-3647. Siciliano, B., Khatib, O. (2008). Springer handbook of robotics.

Swevers, J., Ganseman, C., Bilgin, D., De Schutter, J. and Van Brussel, H. (1997). Optimal robot excitation and identification. IEEE Trans. on Robotics and $\mathrm{Au}$ tomation, Vol. 13, no. 5, 730-740.

Yoshida, K., Nenchev, D.N. and Uchiyama, M. (1995). Moving base robotics and reaction management control. Robotics Research, 100-109. 